\title{
Elección de las plazas de Medicina de Familia en Castilla- La Mancha en las oposiciones MIR de 2011 y 2013
}

\author{
María Candelaria Ayuso Raya ${ }^{\mathrm{a}}$, Eunice de los Santos Berrido ${ }^{\mathrm{b}}$, Samuel Matos Berroac, \\ María Flores Copete ${ }^{\mathrm{b}}$, Ricardo Reolid Martínez ${ }^{\mathrm{b}}$ y Francisco Escobar Rabadán ${ }^{\mathrm{c}}$
}

\begin{abstract}
a Médico de Familia, C.S. de Quintanar del Rey, Albacete. Servicio de Salud de Castilla-La Mancha (SESCAM). ${ }^{\mathrm{b}}$ Residente de Medicina Familiar y Comunitaria. Unidad Docente de Albacete. ${ }^{c}$ Médico de Familia. C.S. Zona IV de Albacete, SESCAM.

Correspondencia:

$\mathrm{M}^{\mathrm{a}}$ Candelaria Ayuso Raya. Centro de Salud Universitario Zona IV, Albacete. Seminario, 4. 02006 Albacete.

Correo electrónico:

candeayuso@hotmail.com

Recibido el 12 de marzo de 2014.

Aceptado para su publicación el 8 de abril de 2014.

Una versión preliminar fue presentada en las I Jornadas de Investigación en Atención Primaria de Castilla-La Mancha.
\end{abstract}

\section{RESUMEN}

Objetivo: Conocer la prioridad concedida a la elección de Medicina de Familia (MF) en CastillaLa Mancha (CLM) entre los opositores MIR 2011 y 2013 que escogieron plaza en esta comunidad, y los factores asociados con esta elección.

Método: Estudio transversal.

Ámbito: Académico.

Participantes: 279 opositores en 2011 y 206 en 2013.

Mediciones y resultados principales: De la web del Ministerio de Sanidad se obtuvo el número en la oposición, el sexo y la especialidad y ciudad elegidas. Se disponía de información adicional de graduados en la Universidad de CLM.

Análisis estadístico: probabilidad de elección de MF relacionada con número de orden en oposición, global y en función de otras variables (Kaplan-Meier).

Resultados: En 2011, MF fue elegida por un 34,4 \%. La mediana del número para elegir MF fue 7.701 (rango intercuartiles [RI]:4.962-10.430), significativamente mayor a otras especialidades $(p<0,0001)$. No existían diferencias por sexo para la elección de MF frente al resto de especialidades. Los de la Universidad de CLM elegían MF con menor número que los demás (medianas: 4.463 y 8.980), aunque en menor proporción ( 13,2 y $37,8 \%$, respectivamente) $(p<0,00001)$. Los resultados correspondientes en 2013 eran: 34 \% eligen MF; mediana: 7.362 (RI: 4.7658.541) $(p<0,0001)$; sin deferencias por sexo; los de la Universidad de CLM elegían MF con menor número (6.704 y 7.622) y menor proporción $(19,05$ y $35,68 \%$, respectivamente) $(p=0,004)$. Conclusión: La elección de plazas de MF en CLM se realiza con números altos de la oposición MIR. Los graduados en Albacete la eligen con mejor número que el resto de opositores, aunque muestran poco interés por ella.

Palabras clave: Educación Médica. Estudios de Grado. Medicina Familiar y Comunitaria.

\section{ABSTRACT}

Choice for family medicine posts in Castilla-La Mancha in the 2011 and 2013 MIR entrance examinations

Objective: To establish priority given to the choice for family medicine (FM) posts in Castilla-La Mancha among MIR 2011 and 2013 candidates who chose this community and factors associated with this choice.

Method: Cross-sectional study.

Scope: Academic.

Participants: 279 candidates in 2011 and 206 in 2013

Main analysed data and results: the following was obtained from the ministry of health website; number of candidates, sex and chosen city and specialization. Additional information on Castilla-La Mancha University graduates was available.

Statistical analysis: likelihood of FM post choice related to candidate order and overall number and was based on other variables (Kaplan-Meier).

Results: In 2011, FM was chosen by $34.4 \%$. The median of the number who chose FM was 7,701 (interquartile range [IQR]: 4.962-10.430), significantly higher than other specializations $(p<0,0001)$. There were no differences in sex for the choice of FM compared to other specializations. Those of the Castilla-La Mancha University opted for FM in lower numbers than the rest (medians: 4.463 and 8.980 ), although in a lower proportion (13.2 and $37.8 \%$ respectively) $(p<0,00001)$.

The corresponding results in 2013 were: $34 \%$ opt for FM; median: 7.362 (RI: 4.765-8.541) $(p<0,0001)$; no differences by sex; those of the Castilla-La Mancha University chose FM in lower number (6.704 and 7.622) and proportion (19.05 y 35.68\%, respectively) ( $p=0,004)$.

Conclusion: The choice of FM posts in Castilla-La Mancha is made in high numbers from the MIR entrance examinations. The graduates in Albacete choose it in higher numbers than other candidates, although they show little interest in it.

Key words: Education, Medical. Undergraduate Studies. Family Practice. 


\section{INTRODUCCIÓN}

Tradicionalmente se ha considerado a la Medicina de Familia (MF) como una de las especialidades que menos despierta el interés de quienes se presentan al examen para médico interno residente (MIR). Por el contrario, son numerosos los informes que constatan que el perfil médico más demandado por el sistema sanitario es el del médico de familia; actualmente representa el $37 \%$ de los médicos del Sistema Nacional de Salud, y las evidencias científicas recomiendan al menos el $50 \%{ }^{1}$. Sin embargo, solo un tercio de la oferta de plazas de MIR en España es para Medicina de Familia y Comunitaria ${ }^{2}$.

La literatura insiste en la crisis generalizada de la atención primaria (AP), que no es un problema exclusivo de España. En Estados Unidos cuesta cubrir las plazas de MF, aunque hay síntomas de una ligera mejoría en $2010^{3}$.

Siguiendo a González López-Valcárcel y Barber Pérez, podemos preguntarnos si lo que está en crisis es el modelo de atención primaria de salud que se inició con la reforma de la década de 1980 en el espíritu de Alma Ata o la especialidad de $\mathrm{MF}^{4}$. Por otra parte, desde hace más de 30 años, MF y universidad caminan conjuntamente, aunque de forma irregular o no bien consolidada. Con anterioridad al Proceso de Convergencia Europea (conocido como Plan Bolonia), de las 29 facultades de Medicina españolas, en tres de ellas la asignatura de MF era obligatoria, en 11 figuraba como asignatura optativa y en tres, como créditos de libre configuración. En 17 facultades había prácticas obligatorias y en 12 , prácticas optativas ${ }^{5}$.

Diversos autores, las sociedades científicas de MF y AP, así como la Comisión Nacional de Medicina Familiar y Comunitaria ${ }^{2,6-8}$, han llamado la atención sobre la falta de continuidad entre la formación de grado y la formación especializada en esta disciplina ${ }^{2}$.

La Facultad de Medicina de Albacete se inauguró en 1998, conforme a las más innovadoras tendencias pedagógicas. En ella se considera el aprendizaje como labor activa del alumno y se sigue un modelo centrado en el estudiante, basado en la integración y resolución de problemas. Se concede una gran importancia a la práctica, orientada a la comunidad y su finalidad es preparar a los estudiantes para las necesidades de la práctica general de la medicina de principios del siglo XXI. En el antiguo plan de estudios existía una asignatura obligatoria de AP en segundo curso ${ }^{9}$, y en el nuevo está prevista una asignatura de MF, también obligatoria, en quinto curso.

El objetivo de este trabajo es describir la prioridad concedida a la elección de la especialidad de MF entre los opositores de las convocatorias MIR de 2011 y 2013 en Castilla-La Mancha (CLM). Así mismo, queremos saber qué factores podrían estar asociados con esta elección.

\section{MATERIAL Y MÉTODOS}

Se trata de un estudio observacional, descriptivo, transversal. Los sujetos de estudio son todos los opositores matriculados en los exámenes MIR de 2011 y 2013 que eligieron plaza de MF en esas convocatorias en CLM (279 y 206, respectivamente). La información fue obtenida de la Web del Ministerio de Sanidad.

Las variables estudiadas han sido: número obtenido en la oposición, sexo, especialidad elegida, ciudad elegida, facultad donde estudiaron (38 y 24 estudiantes de la Facultad de Medicina de Albacete, respectivamente en 2011 y 2013 , que formaban parte de un estudio de cohorte previo).

El análisis estadístico ha consistido en la descripción de frecuencias, análisis no paramétrico (Kruskall-Wallis) y el cálculo de probabilidades de elección de MF en relación con el número de orden en la oposición, global y en función de las otras variables (Kaplan-Meier). Por medio de un análisis de regresión logística, se estudió qué variables eran las que mejor predecían la elección de MF. Se utilizó el paquete estadístico SPSS 12.0.

\section{RESULTADOS}

MF fue elegida por $34,4 \%$ y $34,0 \%$, respectivamente, en 2011 y 2013. En 2011, la mediana del número de orden en la oposición para elegir MF fue 7.701 (IC95 \%: 4.962-10.430), significativamente mayor a otras especialidades $(p<0,0001)$. En esta convocatoria el $57,3 \%$ de los opositores eran mujeres. No existían diferencias por sexo para la elección de MF frente al resto de especialidades, como podemos observar en la figura 1. En la figura 2 queda representado cómo los que habían estudiado en Albacete elegían MF con menor número que los que habían estudiado en otras facultades, con medianas respectivamente de 4.463 (IC95 \%: 3.977-4.949) y 8.980 (IC95 \%: 8.026-9.934), aunque en menor proporción (13,2 y $37,8 \%$, respectivamente) $(p<0,00001)$. 

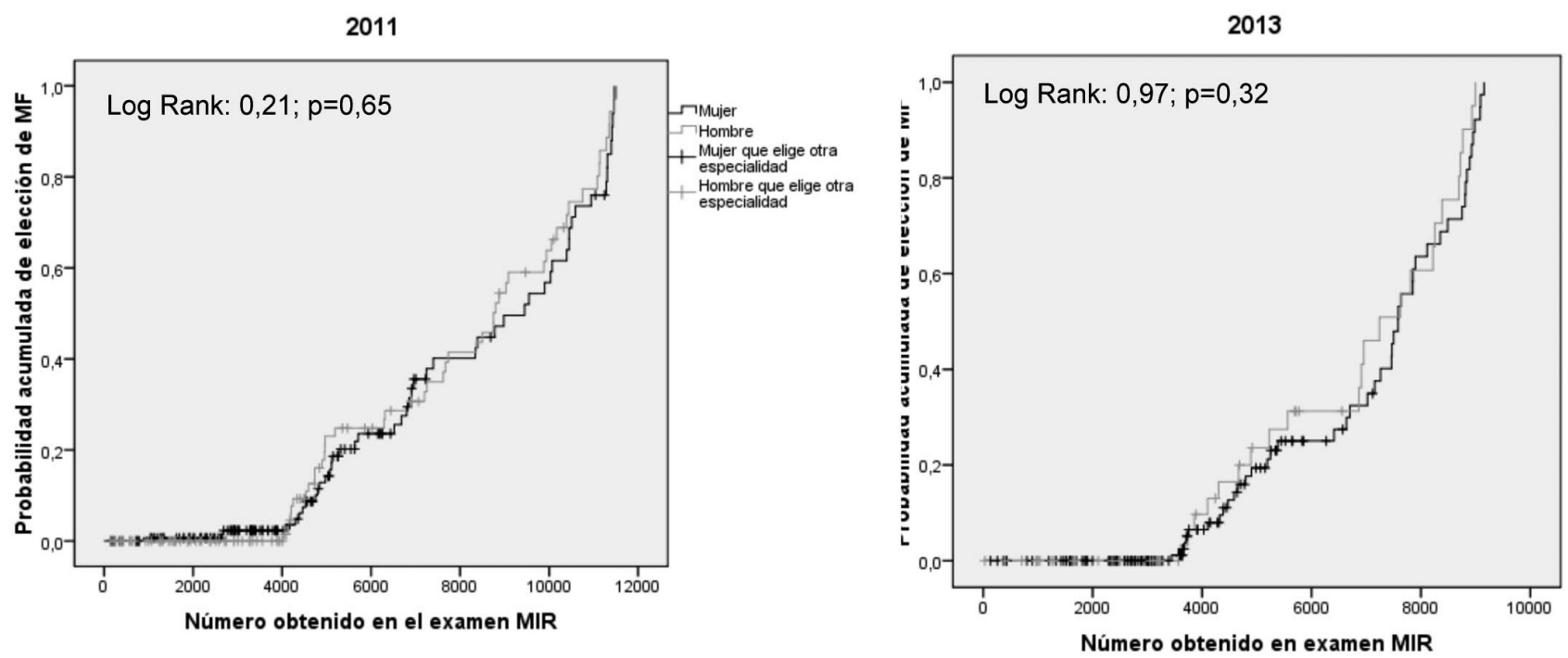

Figura 1. Probabilidad acumulada de elección de Medicina de Familia en función del sexo
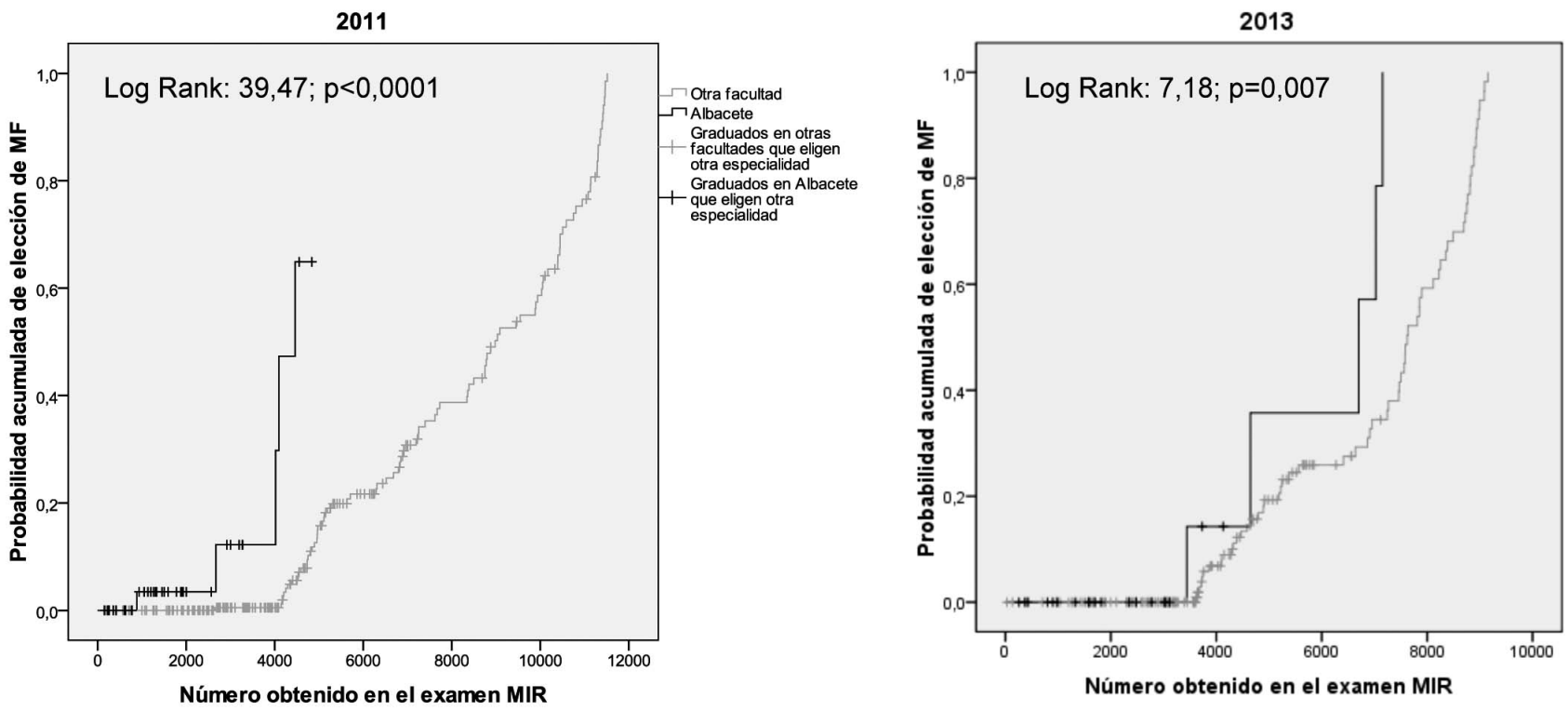

Figura 2. Probabilidad acumulada de elección de Medicina de Familia en función de la Facultad de Medicina donde se graduaron

En 2013 encontramos un aumento en el porcentaje de mujeres entre los opositores que eligen plaza en CLM: $74,1 \%$. Tampoco en esta convocatoria existían diferencias por sexo en cuanto a la elección de especialidad (figura 1). Aunque con diferencias menos marcadas que en 2011 (figura 2), la elección de MF se hizo con menor número: 6.704 (IC95 \%: 3.998-9.410) para estudiantes de la UCLM, frente a 7.622 (IC95 \%: 7.272-7.972) para el resto de facultades, y en menor proporción (19,05 y 35,68 \%, respectivamente) $(p=0,004)$.

En el análisis de regresión logística, solo se encontró asociación significativa de la elección de MF con el número de orden en la oposición (OR: 1,001;
IC95 \%: 1,001-1,001, en ambas convocatorias).

Hemos analizado la elección de otras especialidades, que podríamos considerar de alguna forma relacionadas con la MF o la Atención Primaria. La elección de MF se realizaba con una puntuación significativamente superior a otras especialidades (test de Kruskall-Wallis: $p<0,0001)$, como podemos ver en la figura 3. Era especialmente llamativa la preferencia en especialidades como la ginecología, otras especialidades quirúrgicas y pediatría, con medianas: $2.150,2.170$, y 2.186 , respectivamente, en 2011, y pediatría, especialidades quirúrgicas y ginecología (medianas: $2.140,2.416$ y 2.948 , respectivamente) en 2013. 

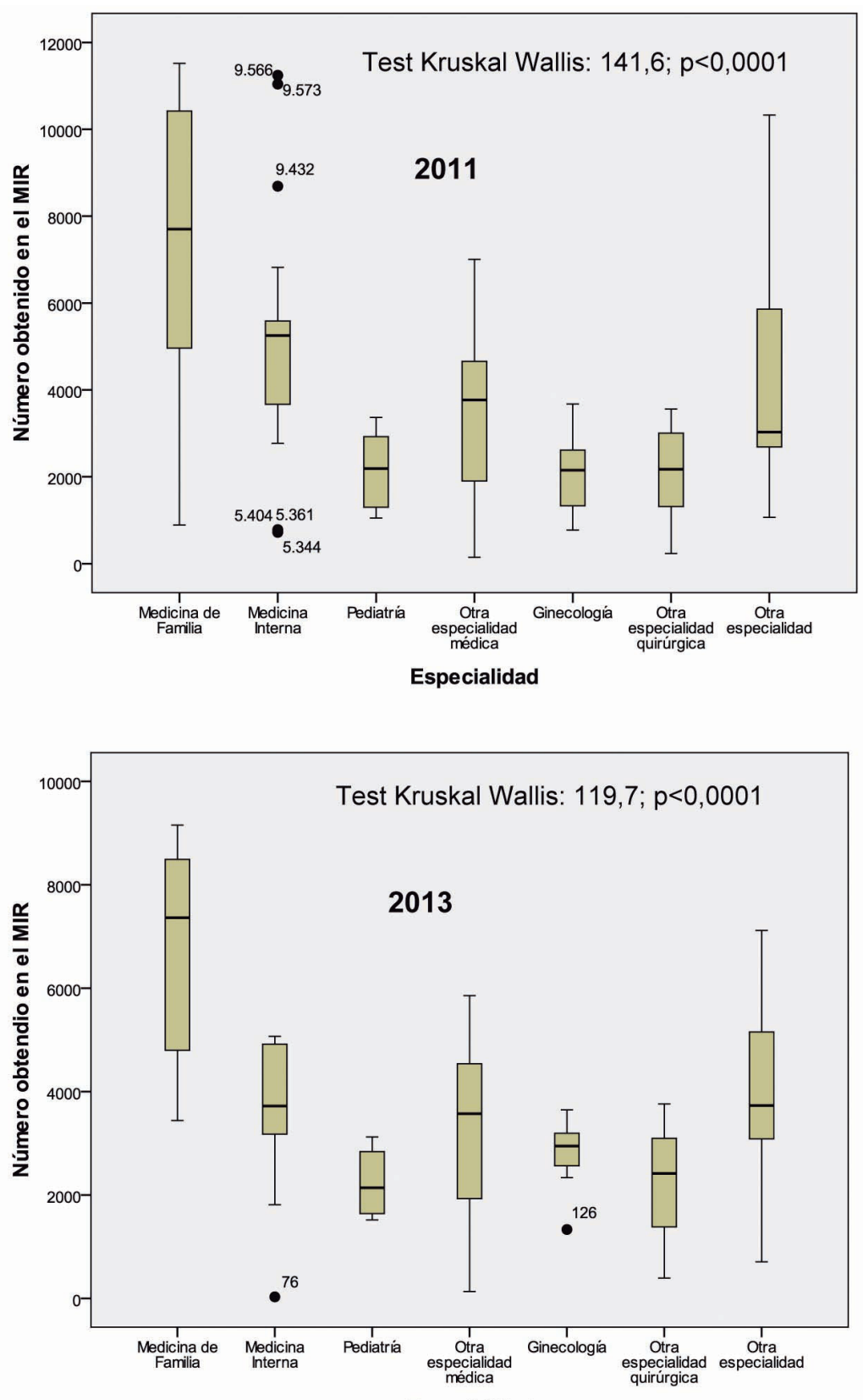

Figura 3. Número de orden en la oposición MIR con el que se eligieron las diferentes especialidades

\section{DISCUSIÓN}

Como hemos señalado al inicio de este trabajo, existe una marcada preocupación por el futuro de la especialidad a tenor del escaso interés que despierta entre los estudiantes de Medicina. Los resultados de nuestro estudio confirman esta apreciación, ya que es en definitiva un número alto en la oposición, con muy pocas posibilidades de optar a otras especialidades, el que determina la elección de MF.

Estos resultados suponen además un verdadero revés para las expectativas que abrió la creación de la Facultad de Medicina en la Universidad de Castilla-La Mancha, con la inclusión de una asignatura obligatoria de Atención Primaria, en tanto en cuanto no ha despertado el interés de los graduados por la especialidad de MF. Por otra parte, son consistentes con los hallazgos a nivel nacional, en el sentido de que la presencia de MF en el currículum no resulta significativa ni se relaciona con el porcentaje de licenciados de las universidades que pudiendo elegir cualquier especialidad escogen $\mathrm{MF}^{10}$.

Las elecciones de especialidad en las oposiciones MIR revelan año tras año las preferencias de 
los jóvenes médicos. Puede resultar chocante que precisamente en la asignación de esas plazas es donde el mercado adquiere un papel predominante dentro del proceso de planificación. Cada candidato elige plaza en orden estricto, según el resultado de la prueba nacional y del expediente académico. El análisis de la posición relativa de las 47 especialidades y de su evolución en el tiempo permite tomar el pulso a ese mercado. Los candidatos MIR tienden a preferir plazas en los hospitales donde han hecho las prácticas clínicas durante el grado 4 .

Existe cierta uniformidad en cuanto a los conocimientos y actitudes hacia la AP y la MF entre los estudiantes de Medicina al principio de sus estudios, incluso independientemente de su interés por estas $^{11}$. Estos conocimientos y actitudes pueden variar en un sentido favorable o desfavorable, dependiendo de las actividades que lleven a cabo a lo largo de su formación en la facultad. En palabras de Allen et al. ${ }^{12}$, cuando las experiencias clínicas en AP han sido positivas a lo largo de los años en la facultad, las actitudes son favorables y hay cambios en las preferencias de la especialidad a favor de la MF.

Es indudable que los conocimientos que adquieran los estudiantes sobre la MF en el pregrado dependen fundamentalmente de la existencia o inexistencia en la facultad de una formación específica en este campo. Al-Dabbagh et al. ${ }^{13}$ evaluaron un modelo de enseñanza de MF basado en tareas y orientado a la comunidad, de cuatro semanas de duración, para un grupo de estudiantes de la Facultad de Medicina de Mosul (Irak) en su último año de formación. Al comparar a este grupo de alumnos con otro cuyos integrantes no habían sido sometidos al aprendizaje en AP, encontraron un incremento estadísticamente significativo, tanto en conocimientos como en destrezas prácticas tras la exposición al modelo. Los estudiantes que participaron mostraron un elevado grado de satisfacción, y consideraron la intervención de gran relevancia para su futuro trabajo. El enfoque sobre habilidades en comunicación, contenidos de la Medicina desde un punto de vista integral y prácticas extrahospitalarias impartidas por profesores motivados en AP son valorados muy positivamente por los estudiantes ${ }^{14,15}$.

La elección de especialidad refleja a lo que han sido expuestos los graduados, las modas y la percepción sobre las especialidades como profesiones sanitarias ${ }^{2}$. Xu et al. ${ }^{16}$, en una encuesta que hicieron a médicos generalistas de Estados Unidos, preguntaban si habían tenido mucho interés por la
AP antes de su formación universitaria y si esa inclinación había variado durante la formación, con especial atención a sus experiencias clínicas en este ámbito asistencial. A partir de las respuestas de 1.561 participantes, encontraron que el $45 \%$ había aumentado su interés por la AP durante su formación de grado; en el 48 \% la inclinación había permanecido constante y en el $7 \%$, había disminuido. Existía una notable asociación entre el cambio de interés por la AP y el hecho de haber seguido cursos clínicos electivos sobre MF en la facultad. Sin embargo, las experiencias clínicas obligatorias en AP no tenían ningún impacto sobre el interés de los estudiantes en seguir especialidades de este nivel de atención. Por tanto, serían los estudiantes que eligen currículos consistentes con sus expectativas o predisposiciones los que cambiarían su inclinación por una carrera generalista, de tal forma que el determinante principal sería el interés por la AP antes de entrar en la facultad de Medicina. Sin embargo, como señalan estos autores, el currículo en la facultad también es importante, en cuanto que proporciona una variedad de opciones para elegir, atendiendo a los intereses individuales de los estudiantes. Por otra parte, era más probable que aquellos alumnos cuyo interés aumentaba durante su formación de grado, se mantuvieran en especialidades de AP diez años después de la graduación, en comparación con los individuos cuyo interés por la misma disminuía. Este es otro indicador de la importancia de la educación médica, que no solo incrementa el interés por la AP de los estudiantes, sino que lo mantiene después de la licenciatura.

En un interesante trabajo publicado por Dornan et al. ${ }^{17}$ se recopilaron los resultados de una serie de estudios hechos entre 1992 y 2001, con el objetivo de analizar experiencias de prácticas en consultas en los primeros años de la licenciatura de medicina, que en un $71 \%$ de los casos tenían lugar en AP / comunidad / MF. Los autores llegaron a la conclusión de que estas experiencias motivaban y satisfacían a los estudiantes y los ayudaban a aclimatarse a los ambientes clínicos, a desarrollarse profesionalmente, a relacionarse con los pacientes con mayor confianza y menos estrés, y a desarrollar la reflexión, destrezas prácticas y una identidad profesional. Asimismo, subrayaban su aprendizaje, haciéndolo más real y relevante para la práctica clínica. Ayudaban a los estudiantes a aprender sobre la estructura y la función del sistema sanitario, y acerca de las actividades preventivas y la función de los profesionales de la salud.

Nieman et al. ${ }^{18}$ hicieron un estudio de cohortes retrospectivo con 267 estudiantes de Medicina que 
eligieron una rotación de cuatro semanas en AP, después de su primer año en la Escuela de Medicina de Houston, entre 2001-2003, y 310 estudiantes que no la eligieron. Los resultados fueron distintos en el desarrollo del examen escrito que integra ciencias y fundamentos de la práctica clínica y en el desarrollo del examen clínico de reestructuración objetiva (OSCE). Los estudiantes que participaron en una de las rotaciones hicieron mejor la OSCE y el examen médico clínico que aquellos que no participaron. Utilizando un análisis de regresión logística, se descartaron posibles variables de confusión, como sexo, rendimiento intelectual previo y conocimientos adquiridos antes de la rotación.

McKnee et al. ${ }^{19}$ evaluaron el impacto de un programa diseñado en 2001 para proporcionar a los estudiantes de Medicina información acerca del desafío y la gratificación que supone optar por una carrera de MF. En el año 2006 analizaron los resultados, llegando a la conclusión de que los estudiantes interesados en la MF reforzaban su decisión a la hora de elegir la especialidad.

Martín Zurro et al. ${ }^{20}$ diseñaron un cuestionario autoadministrado de 70 ítems (más 13 específicos para $3^{\circ}$ y $5^{\circ}$ ) con 6 opciones de respuesta en una escala tipo Likert. El cuestionario anónimo incluía preguntas sobre aspectos sociodemográficos y académicos. Realizaron dos cortes transversales en los cursos 2009-2010 y 2011-2012, obteniendo información de 5.299 y 3.869 alumnos respectivamente, incluyendo estudiantes de primero, tercero y quinto de 22 facultades de medicina que participaron (de las 27 españolas). Solo el $25 \%$ de los estudiantes de primer año tenían claras preferencias por una especialización, aumentando moderadamente el interés por la MF a lo largo de los años de la licenciatura. Prefieren las especialidades médicas hospitalarias (88-89\%) seguidas de las quirúrgicas y la pediatría, resultados similares a los obtenidos en nuestro estudio. El interés por la MF aumentaba con los años de estudio $(36,7 \%$, $41,7 \%$ y $50,2 \%$ en $1^{\circ}, 3^{\circ}$ y $5^{\circ}$ respectivamente; $\mathrm{p}<0,001)$, independientemente de las características de los estudiantes o la facultad a la que asistieran. Entre el tercer y el quinto año, un 54,6\% de los alumnos decían que sus preferencias de especialidad habían cambiado durante los años de facultad y el 83,3\% que las rotaciones en centros de AP eran útiles. Los estudiantes perciben la MF y AP como un ámbito esencial del sistema sanitario y de ejercicio profesional pero con escaso atractivo científico-técnico. El $87 \%$ consideran necesaria y obligatoria la formación en MF y que debería iniciarse en el tercer curso. Al iniciar la carrera so- lamente entre el 24 y el $28 \%$ de los estudiantes tienen una decisión clara sobre la especialidad que van a escoger ${ }^{21}$.

Como conclusión, podemos subrayar que la elección de plazas de MF en CLM se realiza con números altos de la oposición MIR. Los graduados en la UCLM eligen esta especialidad con mejores puestos que el resto de opositores, aunque no es una opción preferencial en su elección. Estos estudiantes de la UCLM habían tenido una asignatura obligatoria de AP en el segundo año de su currículum. Con los nuevos planes de estudio está previsto que se imparta en quinto curso del grado. Esto nos va a permitir valorar los posibles cambios que se produzcan en el futuro.

\section{BIBLIOGRAFÍA}

1. Starfield B, Shi L, Macinko J. Contribution of primary care to health systems and health. The Milbank Quartely. 2005;83:457-502.

2. Casado Vicente v, Bonal Pitz P, Cucalón Arenal JM, Serrano Ferrández E, Suárez Gonzalez F. La medicina familiar y comunitaria y la universidad. Informe SESPAS 2012. Gac Sanit. 2012;26(S):69-75.

3. Cooke M, Irby DM, O'Brien BC. Educating physicians: a call for reform of medical school and residency. Stanford: The Carnegie Foundation for the Advancement of Teaching; 2010.

4. González López-Valcárcel B, Barber Pérez P. Planificación y formación de profesionales sanitarios, con foco en la atención primaria. Informe SESPAS 2012. Gac Sanit. 2012;26(S):46-51.

5. Bonal Pitz P, Casado Vicente V, Cots Yago JM, Monreal Hijar A, Gil Guillén V, Calvo Hernando E. La medicina de familia como área de conocimiento (VII): presencia de la medicina de familia en las facultades de medicina de España. Tribuna Docente. 2005;6:3-13.

6. Bonal P. Problemas y disfunciones en la formación MIR en medicina de familia y comunitaria generadas por las características actuales de la licenciatura de medicina. Tribuna Docente. 2007;8:1-6.

7. Buddeberg-Fischer B, Klaghofer R, Abel T, Buddeberg C. Swiss residents' speciality choices--impact of gender, personality traits, career motivation and life goals. BMC Health Serv Res. 2006;6:137.

8. Kassebaum DG, Szenas PL, Schubert MK. Determinants of the generalist career intentions of 1995 graduating medical students. Acad Med. 1996;71:197-209.

9. Fernández C, López-Torres J, Escobar F, Montoya J, Garcia A. Aprendizaje de la Medicina de Familia en la Facultad de Medicina en Castilla-La Mancha. Tribuna Docente 2005; 6:3-4.

10. González Lopez-Valcarcel B, Ortún V, Barber P, Harris JE. Importantes diferencias entre facultades de medicina. Implicaciones para la medicina de familia y comunitaria. Aten Primaria. 2014;46:140-6.

11. Grayson MS, Newton DA, Whitley TW. First-year medical students' knowledge of and attitudes toward primary care careers. Fam Med. 1996;28:337-342. 
12. Allen SS, Sherman MB, Bland CJ, Fiola JA. Effect of early exposure to family medicine on students' attitudes towards the specialty. J Med Educ. 1987;62:911-917.

13. Al-Dabbagh SA, Al-Taee WG. Evaluation of a task-based community oriented teaching model in family medicine for undergraduate medical students in Iraq. BMC Med Educ. 2005;5:31.

14. Matson CC, Ullian JA, Boisaubin EV. Integrating early clinical experience curricula at two medical schools: lessons learned from The Robert Wood Johnson Foundation's Generalist Physician Initiative. Acad Med. 1999;74:53-58.

15. Miettola J, Mäntyselkä P, Vaskilampi T. Doctor-patient interaction in Finnish primary health care as perceived by first year medical students. BMC Med Educ. 2005;5:34.

16. Xu G, Hojat M, Brihgam TP, Veloski JJ. Factors associated with changing levels of interest in primare care during Medical School . Academic. 1999;74:1011-1015.

17. Dornan T, Littlewood S, Margolis SA, Scherpbier A, Spencer J, Ypinazar V. How can experience in clinical and community settings contribute to early medical education? A BEME systematic review. Med Teach. 2006;28:3-18.
18. Nieman LZ, Cheng L, Hormann M, Farnie MA, Molony DA, Butler B. The impact of preclinical preceptorship on learning the fundamentals of clinical medicine and physical diagnosis skills. Acad Med. 2006;81:342-346.

19. McKee ND, McKague MA, Ramsden VR, Poole RE. Cultivating interest in family medicine. Family medicine interest group reaches undergraduate medical students. Can Fam Physician. 2007;53:661-665.

20. Martín Zurro A, Jiménez Villa J, Monreal Hijar A, Mundet Tuduri X, Otero Puime A, Alonso-Coello P and for the Universidad y Medicina de Familia (UNIMEDFAM) research group. Medical student attitudes towards family medicine in Spain: a statewide analysis. BMC Fam Pract.2012;13:47.

21. Martín Zurro A, Jiménez Villa J, Monreal Hijar A, Mundet Tudurid X, Otero Puime A, Alonso Coello P, por el Grupo de Investigación Universidad y Medicina de Familia (UNIMEDFAM). Los estudiantes de medicina españoles y la medicina de familia. Datos de las 2 fases de una encuesta estatal. Aten Primaria. 2013;45:38-45. 